\title{
Autonomous Voltage Security Regions to Prevent Cascading Trip Faults in Wind Turbine Generators
}

Niu, Tao; Guo, Qinglai; Sun, Hongbin; Wu, Qiuwei; Zhang, Boming; Ding, Tao

Published in:

IEEE Transactions on Sustainable Energy

Link to article, DOI:

10.1109/TSTE.2016.2545704

Publication date:

2016

Document Version

Peer reviewed version

Link back to DTU Orbit

Citation (APA):

Niu, T., Guo, Q., Sun, H., Wu, Q., Zhang, B., \& Ding, T. (2016). Autonomous Voltage Security Regions to Prevent Cascading Trip Faults in Wind Turbine Generators. IEEE Transactions on Sustainable Energy, 7(3), 1306 - 1316. https://doi.org/10.1109/TSTE.2016.2545704

\section{General rights}

Copyright and moral rights for the publications made accessible in the public portal are retained by the authors and/or other copyright owners and it is a condition of accessing publications that users recognise and abide by the legal requirements associated with these rights.

- Users may download and print one copy of any publication from the public portal for the purpose of private study or research.

- You may not further distribute the material or use it for any profit-making activity or commercial gain

- You may freely distribute the URL identifying the publication in the public portal 


\title{
Autonomous Voltage Security Regions to Prevent Cascading Trip Faults in Wind Turbine Generators
}

\author{
Tao Niu, Qinglai Guo, Senior Member, IEEE, Hongbin Sun, Senior Member, IEEE \\ Qiuwei Wu, Senior Member, IEEE, Boming Zhang, Fellow, IEEE, Tao Ding, Member, IEEE
}

\begin{abstract}
Cascading trip faults in large-scale wind power centralized integration areas bring new challenges to the secure operation of power systems. In order to deal with the complexity of voltage security regions and the computation difficulty, this paper proposes an autonomous voltage security region (AVSR) for each wind farm and the point of common coupling (PCC) substation, whose voltage can be controlled in a decoupled way. The computation of the AVSR can be completed using a stepwise search method exchanging voltage and power information between the control center and the wind farms. At each wind farm, an AVSR is determined to guarantee the normal operation of each wind turbine generator (WTG), while in the control center, each region is designed in order to guarantee secure operation both under normal conditions and after an $N-1$ contingency. A real system in Northern China was used to carry out case studies to verify the effectiveness of the AVSRs proposed, and good performance was demonstrated using the Monte Carlo method.
\end{abstract}

Index Terms - autonomous voltage security region (AVSR), $N-1$ contingency, voltage control, wind power integration

\section{NOMENCLATURE}

$\left[\frac{V_{P O C, i}^{A V S R}, \overline{V_{P O C, i}^{A V S R}}}{\left[\frac{V_{P C C}^{A V S R}}{V_{P C C}^{A V S R}}\right]}\right.$
$n_{i}$
$N_{w}$
$N_{g}$
$N_{s}$
$p_{w, i, j}^{0}, q_{w, i, j}^{0}$
$v_{w, i, j}^{0}$
$\Delta Q_{i}^{0}$

The autonomous voltage security region (AVSR) of point of coupling (POC) bus in wind farm $i$

The AVSR of point of common coupling (PCC) bus

Number of wind units in wind farm $i$

Number of wind farms

Number of conventional generators

Number of scenarios

Active and reactive power of wind unit $j$ in wind farm $i$ under normal conditions

Voltage magnitude of wind unit $j$ in wind farm $i$ under normal conditions

Total reactive power regulation of wind

Manuscript received October 19, 2015; revised February 27, 2016; accepted March 19, 2016. This work was supported in part by National Natural Science Foundation of China $(51277105,51522702)$ and National Key Basic Research Program of China (2013CB228206). Paper no. TSTE-00866-2015.

Tao Niu, Qinglai Guo, Hongbin Sun and Boming Zhang are with the Department of Electrical Engineering, Tsinghua University, Beijing, China. (email: guoqinglai@tsinghua.edu.cn)

Qiuwei Wu is with Technical University of Denmark, Department of Electrical Engineering, Elektrovej 325 Kgs. Lyngby, DK 2800

Tao Ding is with Xi'an Jiaotong University, Department of Electrical Engineering, No.28, Xianning West Road, Xi'an, Shanxi, CN 710049 farm $i$ under normal conditions (The superscript 0 denotes normal conditions)

Total reactive power regulation of conven-

$$
\begin{gathered}
\Delta Q_{g, m}^{0} \\
\Delta Q_{s v c, i}^{s} \\
V_{P O C, i}^{0}, V_{P O C, i}^{s} \\
V_{P C C}^{0}, V_{P C C}^{s}
\end{gathered}
$$

tional power plant $m$ in the control center under normal conditions

Total reactive power regulation of static voltage compensators (SVCs) in wind farm $i$ in scenario $s$

Voltage magnitude of POC bus in wind farm $i$ under normal conditions and in scenario $s$

Voltage magnitude of PCC bus under normal conditions and in scenario $s$

\section{INTRODUCTION}

A $S$ the most promising renewable energy source (RES), wind power is widely used over the world. There are several voltage-related challenges for accommodating large-scale wind power such as voltage fluctuations and the voltage stability under disturbances. In order to address these operation issues in wind power grid, a number of techniques have been developed to enhance the wind power hosting capacity and stability of the power system [1-5], maintain the voltage of the wind power integration area within limits [6-11] and maintain an appropriate voltage profile with the help of on-load tap changes (OLTCs) and capacitor/reactor banks [25-27]. Furthermore, static methods such as PV curves and continuation power flow (CPF) are used to analyze the risk of voltage instability from the perspective of voltage stability in wind systems. There are also some researches focus on improving the voltage stability [12-14] of the power system with wind power.

One of the major challenges for large-scale wind power in China is cascading trip faults. During 2011 2014, several severe cascading trip incidents occurred in China due to the normal but not safe operation state [19]. Once a trip fault occurs, other wind farms' voltage will significantly increase and cannot be kept within a normal voltage limit. As a consequence, wind turbine generators (WTGs) in other wind farms are tripped by high-voltage protection systems. As more WTGs trip, wind farm voltages become higher, resulting in cascading trip faults. Here, a cascading trip incident in Zhangbei Wind Power Base in Northern China on Feb 26 $6^{\text {th }}$ 2012, was recorded in Fig. 1 by synchronized measurements from deployed phasor measurement units (PMUs). As shown in Fig. 1, the cascading faults were triggered by short-circuit faults in wind farm GT, which 
caused the very low voltage. Unfortunately, most of the WTGs in China were not equipped with effective low-voltage ride through (LVRT) control, so these WTGs were shut down. Combined with the capacitors that were not switched off in time, this led to a sudden large amount of redundant reactive power. Due to the fact that the wind power pool area was connected with a relatively weak power grid, afterwards, the voltage profile in this integration area significantly increases during $0.4 \mathrm{~s} \sim 2.0 \mathrm{~s}$, resulting in great wind generation loss. According to the data from State Electricity Regulatory Commission of China, there are 193 large-scale cascading trip incidents during January to August in 2011 in Northern China, and the most severe wind power loss in an incident was $500 \mathrm{MW}$, which brings great challenges to power system operation.

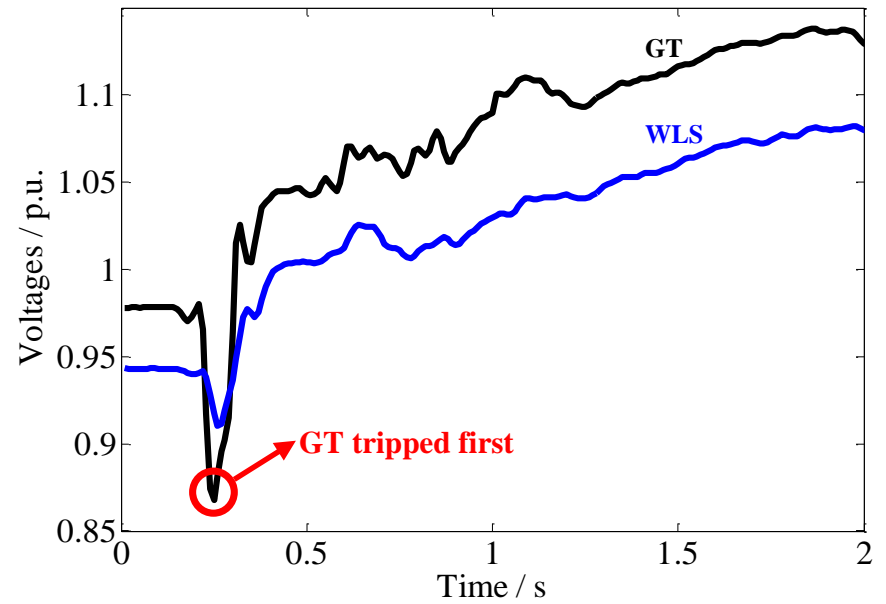

Fig. 1. Typical trip-off process voltage during a cascading failure

We can conclude from the cascading trip process that the static voltage profiles are crucial to secure operations and the most important reason leading to cascading trip is the improper static voltage magnitude profiles.

Thus in order to deal with the large-scale cascading trip problems and keep the wind farms working under normal and safe state, voltage security region is then proposed to determine voltage operation ranges for all the important buses in the wind pool area. When the wind farms operate within these regions, once a wind farm trips, other wind farms can still operate at an acceptable voltage level, and will not lead to cascading trip. There is some preliminary research on static voltage security region [15-17], however, some challenges still remain.

Firstly, large-scale wind pool areas usually include dozens of wind farms with thousands of WTGs, and all the voltage of the area shall be taken into account. Otherwise, any trip incident may result in cascading trip faults. A control center can hardly model all the details to guarantee each WTG's operation constraints both under normal conditions and $\mathrm{N}-1$ contingencies.

Secondly, the boundary of static voltage security region is complex due to the intermittent and stochastic characteristics of wind power. Therefore, the computation of voltage security region for online application is also a great challenge. Several studies have proposed methods for reducing the computational burden of the boundary. Based on the two-level wind automatic voltage control (AVC) system in [18-19], an approximate $N-1$ voltage security region boundary encompassed by cutting planes for centralized multiple wind farms is presented in [20] and [21]. However, with the linearization assumption, the accuracy is sacrificed to reduce the computation burden.

Last, but not least, for practical applications, the hierarchical wind-AVC system uses an autonomous voltage controller in each wind farm and a synergic voltage controller in the control center [19]. It is more promising and practical for wind farms to independently control themselves without considering the details of other wind farms' operation. The previous research [22] didn't produce decoupled voltage operation ranges for wind farms.

Therefore, a concept of AVSR (autonomous voltage security region) is proposed in this paper. The definition of the AVSR in the wind power grids is: If the control center controls the point of common coupling (PCC) bus and wind farms control their own point of coupling (POC) buses in their own certain ranges, each wind farm can control all the WTGs within normal operation ranges under both normal conditions and any $\mathrm{N}-1$ contingency, without considering the operation details of other wind farms. The set of these voltage operation ranges for PCC bus and POC buses is defined as the AVSR. The concept of "autonomous" means each wind farm can control their voltages by themselves without considering other wind farms, i.e., the AVSR produces decoupled voltage control strategies for each wind farm. The AVSR in this paper is proposed from the perspective of security, aims to deal with the large-scale cascading trip faults caused by high-voltage protection systems of WTGs when the terminal voltages of WTGs exceed their upper bounds after an N-1 contingency. According to the analysis of cascading trip process in Fig. 1, we can conclude that the how to compute autonomous voltage security region (AVSR) to acquire voltage security control ranges of each wind farm and avoid cascading trip is a static voltage security problem, and the computation of AVSR should be based on static power flow equations. The ASVR computed here could be adopted as constraints for the voltage controllers that are deployed in wind farms and control center. During the control process, of course, the dynamic models are crucial, especially the dynamic reactive power reserve of SVCs/SVGs will greatly influence the voltage profiles when contingencies happen [30]-[31]. In future work, we will further research how dynamics models influence the AVSR (e.g. the optimal allocation of SVC/SVGs) in wind farms.

This paper aims to extend the previous work to propose the static AVSR and focus on accelerating the computation. And the contributions of the paper can be summarized as follows.

(1) The AVSR for the PCC bus and the POC bus in each wind farm is proposed and can be used for decoupled voltage control among all wind farms and the PCC bus. That's the most important contribution.

(2) In previous studies [22], an iterative method for the system-wide computation was proposed. The stepwise search method proposed in this paper does not require iteration, and the necessary constraint information is exchanged only once between the control center and each wind farm, resulting in less necessary computation time. 
(3) The detailed networks of each wind farm are taken into account, and a complete power flow model is used instead of a traditional sensitive model in each wind farm. Thus the results are more accurate.

(4) DistFlow format [23] is used to compute optimal power flow (OPF) in each wind farm. This model is completely equivalent to power flow model with polar coordinates format in radial networks [23], which guarantees the computation accuracy. The stepwise method proposed in this work needs repeated computation, and the DistFlow model significantly saves computation time due to the higher linearity.

The paper is organized as follows. The voltage feasible region (VFR) for an individual wind farm is first described in Section II. Based on these VFRs, the AVSR is computed. Six necessary steps to acquire the AVSR and the information exchange between wind farms and the control center are given at the end of Section II. Section III presents case studies of a simple system and a real system. The effectiveness of the proposed stepwise method is demonstrated by Monte Carlo simulation in Section III followed by conclusions.

\section{AVSR IN WIND POOL AREA}

\section{A. Computation structure of AVSR}

Unlike hydro and thermal power plants, wind farms are often distributed over large areas and the wind powers are then connected to a high-voltage bus $(110 / 220 \mathrm{kV}$-level) via several feeders (35 kV-level) in wind farms. The high-voltage bus in each wind farm is the POC bus. Several wind farms are then connected to a PCC bus in a substation at a higher voltage level $(220 / 500 \mathrm{kV})$ via transmission lines, then centrally integrated into the power grid. A typical structure for centralized integration of wind farms in Northern China is shown in Fig. 2.

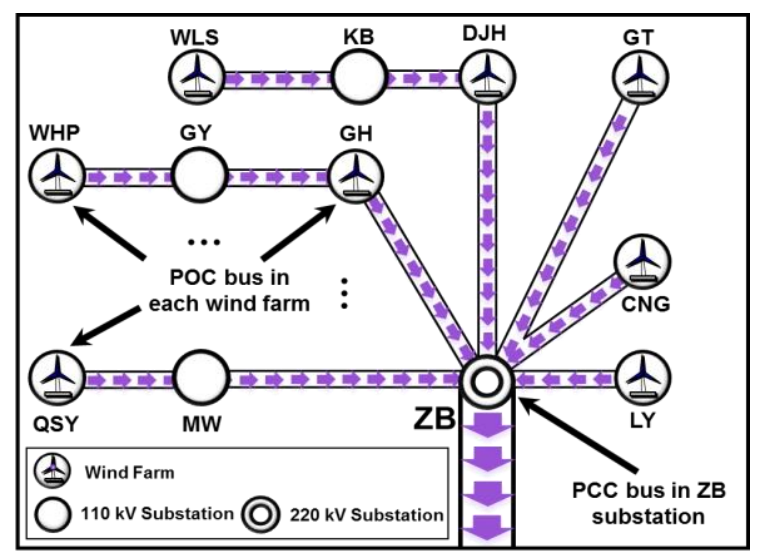

Fig. 2. Typical structure for centralized integration of wind farms in Northern China

According to the hierarchical wind-AVC system [19], the voltage of the PCC bus is controlled by the control center while the voltage of POC bus is controlled by wind farms. Therefore, it is feasible to find a decoupled voltage control range (the AVSR) for the control center and each wind farm. According to the AVC system, the AVSR's computing structure is designed as follows.

For each wind farm, an autonomous control strategy is designed to keep the POC bus within their own range, which takes into account the wind farm's parameters and aims to acquire both maximum and minimum voltage magnitudes for each POC bus in a centralized integration area.

For the control center, a synergistic control strategy is designed to coordinate all distributed POC buses, which uses the security-constrained optimal power flow (SCOPF) and ensures that the voltage of all wind farms satisfies the operational constraints under both normal conditions and after an $\mathrm{N}-1$ contingency.

\section{B. VFR for an individual wind farm}

The typical structure of an individual wind farm is shown in Fig. 3. In each wind farm, the detailed network topology of different kinds of devices is taken into account. First, for an individual wind farm, it is supposed to find the VFR for the POC bus $\left(V_{P O C, i}^{0}\right)$ and the PCC $\left(V_{P C C}^{0}\right)$ bus.

Note that the voltage of each WTG in a wind farm is a state variable, and the reactive power of each device is a control variable. In each wind farm, for a specified active power (1-f) and the given voltage of $V_{P C C}^{0}(1-\mathrm{g})$, we only use the reactive power control capability of its own wind farm (1-d) to prevent all WTGs from tripping-off (1-e). The cascading trip incidents may happen if (1-e) is violated. The maximum and minimum value of $V_{P O C, i}^{0}(1-\mathrm{a}) \sim(1-\mathrm{b})$ are supposed to be obtained for voltage control of the wind farm. When $V_{P O C, i}^{0}$ operate within $\left[V_{P O C, i}^{0}, \overline{V_{P O C, i}^{0}}\right]$, all WTGs can operate normally only by using the control capability of the wind farm.

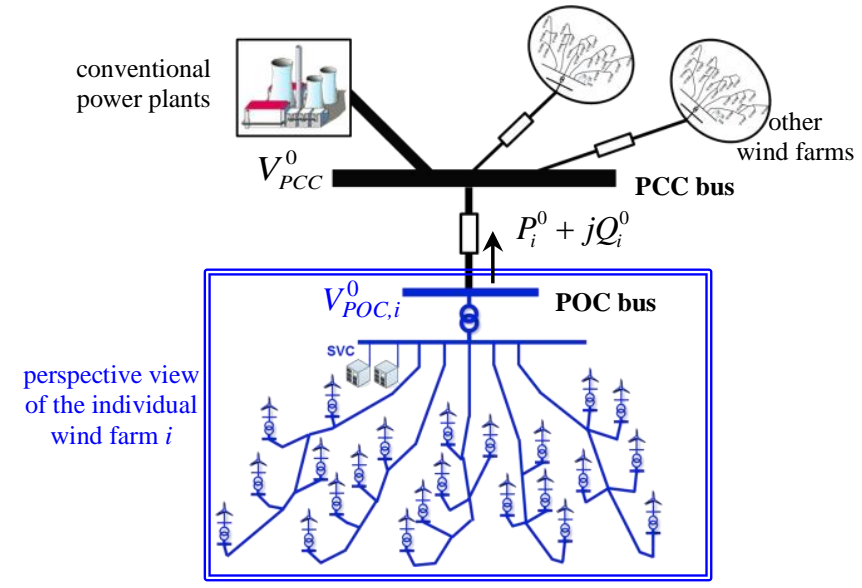

Fig. 3. Perspective view of an individual wind farm

$$
\begin{gathered}
\frac{V_{P O C, i}^{0}}{\overline{V_{P O C, i}^{0}}}=\min _{q_{w, i, j}^{0}} V_{P O C, i}^{0}{ }_{q_{w, i, j}^{0}} V_{P O C, i}^{0} \\
\text { s.t. } \quad \boldsymbol{f}_{w}^{0}\left(\boldsymbol{p}_{i}, \boldsymbol{q}_{i}, \boldsymbol{p}_{i}^{l}, \boldsymbol{q}_{i}^{l}, \boldsymbol{v}_{i}, \boldsymbol{I}_{i}\right)=0 \\
\frac{q_{w, i, j}^{0}}{\overline{v_{w, i, j}^{0}}} \leq q_{w, i, j}^{0} \leq v_{w, i, j}^{0} \leq \overline{q_{w, i, j}^{0}} \\
p_{w, i, j}^{0}=p_{w, i, j}^{S P} \\
V_{P C C}^{0}=V_{P C C}^{0, S P} \\
j=1,2, \ldots, n_{i}
\end{gathered}
$$


The radial network feature of wind farms is fully considered to improve computation performance. Here, (1-c) expresses the power flow model in DistFlow [24] format, which include three linear constraints (1-c-1) (1-c-3) and one quadratic constraint (1-c-4). The higher linearity of the DistFlow model results in considerable time saving for the stepwise method below with repeated computation [23].

$$
\begin{gathered}
p_{i, j+1}^{b}=p_{i, j}^{b}-I_{i, j}^{2} r_{i, j}-p_{i, j+1}-v_{i, j}^{2} G_{i, j} \\
q_{i, j+1}^{b}=q_{i, j}^{b}-I_{i, j}^{2} x_{i, j}-q_{i, j+1}-v_{i, j}^{2} B_{i, j} \\
v_{i, j+1}^{2}=v_{i, j}^{2}-2\left(r_{i, j} p_{i, j}^{b}+x_{i, j} q_{i, j}^{b}\right)+I_{i, j}^{2}\left(r_{i, j}^{2}+x_{i, j}^{2}\right) \\
p_{i, j}^{b{ }^{2}}+q_{i, j}^{b 2}=v_{i, j}^{2} I_{i, j}^{2}
\end{gathered}
$$

In wind farm $i, p_{i, j}^{b}$ and $q_{i, j}^{b}$ denote active and reactive power of branch $j, p_{i, j}$ and $q_{i, j}$ denote active and reactive power load of node $j, I_{i, j}$ denotes the current of branch $j$.

Actually, $V_{P C C}^{0}$ is controlled by the control center and not fixed. So from the wind farm side, let $V_{P C C}^{0}$ increase and decrease respectively stepwise by 0.01 p.u. from current value, the corresponding $V_{P O C, i}^{0}$ and $\overline{V_{P O C, i}^{0}}$ are computed for each given $V_{P C C}^{0, S P}$ by solving (1), and these solution points are combined together to acquire the maximum and minimum curves for $V_{P O C, i}^{0}$, as shown in Fig. 4. The region bounded by the two curves is the VFR of an individual wind farm, which is the set of all feasible operational points, and guarantees normal operation of all WTGs under normal conditions.

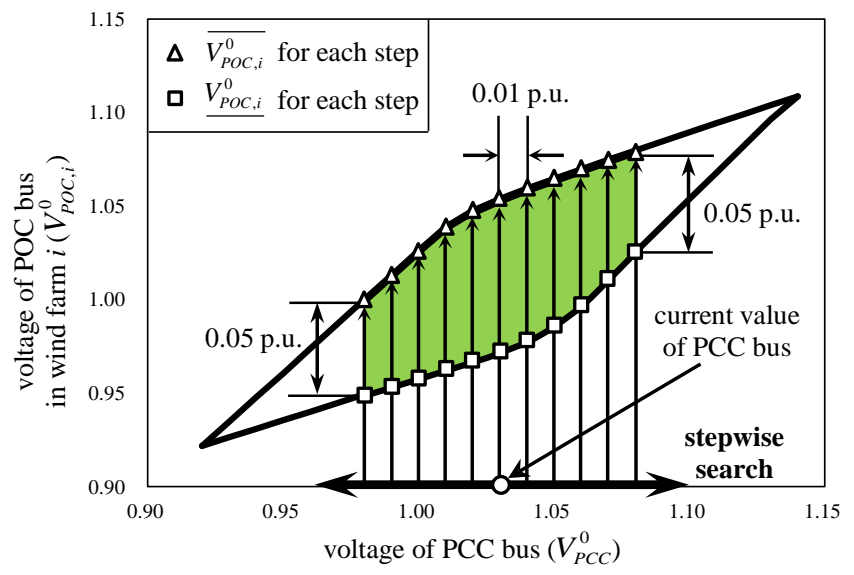

Fig. 4. Stepwise search method in an individual wind farm

It should be noted that:

1) Decreasing the search step will produce more accurate results, but this will lead to a significant increase in computation time. Considering both accuracy and efficiency, search step is set as 0.01 p.u.

2) For the given $V_{P C C}^{0, S P}$, it is not practical for wind-AVC if the voltage operation range $\left[V_{P O C, i}^{0}, \overline{V_{P O C, i}^{0}}\right]$ is too small. Considering both computation amount and practicability, the stepwise search ends when the voltage operation range $\left[V_{P O C, i}^{0}, \overline{V_{P O C, i}^{0}}\right]$ is less than 0.05 p.u., as shown in Fig. 4, the green region will be used as the VFR for each individual wind farm.
3) For each step, once $V_{P C C}^{0, S P}$ is given, $V_{P O C, i}^{0}$ is increasing in wind farm's total reactive power $Q_{i}^{0}$ (in Fig. 3). Thus for each given $V_{P C C}^{0, S P}$, wind farm's corresponding total reactive control capability $Q_{i}^{0}$ and $\overline{Q_{i}^{0}}$, which are used below for system-side in (3), can be obtained from optimal solutions of $V_{P O C, i}^{0}$ and $\overline{V_{P O C, i}^{0}}$ respectively by solving (1).

4) On-load tap changers (OLTCs) and capacitor/reactor banks are not considered as optimal variables due to the following two reasons: First, in China, most tap changers in wind farms could not be online regulated in operation. Few OLTCs are used to optimize the voltage profile only 3 5 times a day. In terms of capacitor/reactor banks, one of the reason leading to the cascading trips is the improper switches capacitor/reactor banks, which are gradually replaced by SVC/SVGs in wind farms in China. Thus this paper mainly focuses on the coordination of WTGs (wind turbine generators) and SVC/SVGs. Second, in order to guarantee secure and economic operations of wind farms in China, some wind farms may use the OLTCs and capacitor/reactor banks to optimize the voltage scheme every 1 4 hours. But different from OLTCs and capacitor/reactor banks' optimization, the proposed AVSR is applied to real wind systems online for 1 5 minutes' level. Therefore, the control center will refresh the AVSR after every operation of the OLTCs and capacitor/reactor banks. When wind farm computes AVSR, the operation state of OLTCs and capacitor/reactor banks remains unchanged, thus they are not considered as optimal variables in AVSR computation model.

5) Due to the fact that the computation time of AVSR is always less than 20 seconds and the AVSR is applied to real wind systems for $1 \sim 5 \mathrm{mins}$ level, the computation of AVSR is based on the assumption that a short-term wind speed forecasting model is known with sufficient accuracy [28]-[29]. Thus the AVSR model uses the current active power interface. Thus the AVSR model uses the current active power interface. On the other hand, the control center will refresh the AVSR every 1 5mins online in wind operation, which also guarantees the accuracy of AVSR in a real wind system.

\section{AVSR for multiple wind farms}

Based on the VFRs for each individual wind farm above, the AVSR is then proposed for POC buses of all wind farms and the PCC bus in the control center.

The AVSR means: each wind farm can autonomously control all their WTGs within normal operation ranges not only under normal conditions but also under any $N$-1 contingency (without considering the operation details of other wind farms) in a decoupled way as long as the control center controls $V_{P C C}^{0}$

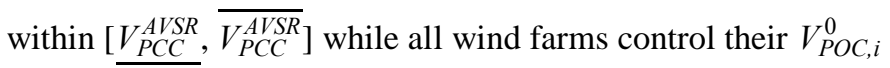
within $\left[V_{P O C, i}^{A V S R}, \overline{V_{P O C, i}^{A V S R}}\right]$.

Obviously, the irregularly-shaped VFR (green regions) in Fig. 5 cannot be used for decoupled voltage control between 
PCC bus and each wind farm because the operation range for $V_{P O C, i}^{0}$ depends on the value of $V_{P C C}^{0}$. Thus, the rectangle-shaped regions can be used for decoupled control because the operation range for $V_{P O C, i}^{0}$ is independent of $V_{P C C}^{0}$. (operation range for $V_{P O C, i}^{0}$ remains unchanged when $V_{P C C}^{0}$ changes, i.e., in the blue rectangles, the voltage operation range for $V_{P O C, i}^{0}$ is always $\left[V_{P O C, i}^{A V S R}, \overline{V_{P O C, i}^{A V S R}}\right]$ as long as the voltage of $V_{P C C}^{0}$ remains within $\left.\left[\overline{V_{P C C}^{A V S R}}, \overline{V_{P C C}^{A V S R}}\right]\right)$. As shown in Fig. 5, the blue rectangles in each figure are the AVSR, which can be used for decoupled control between the PCC bus and each wind farm.

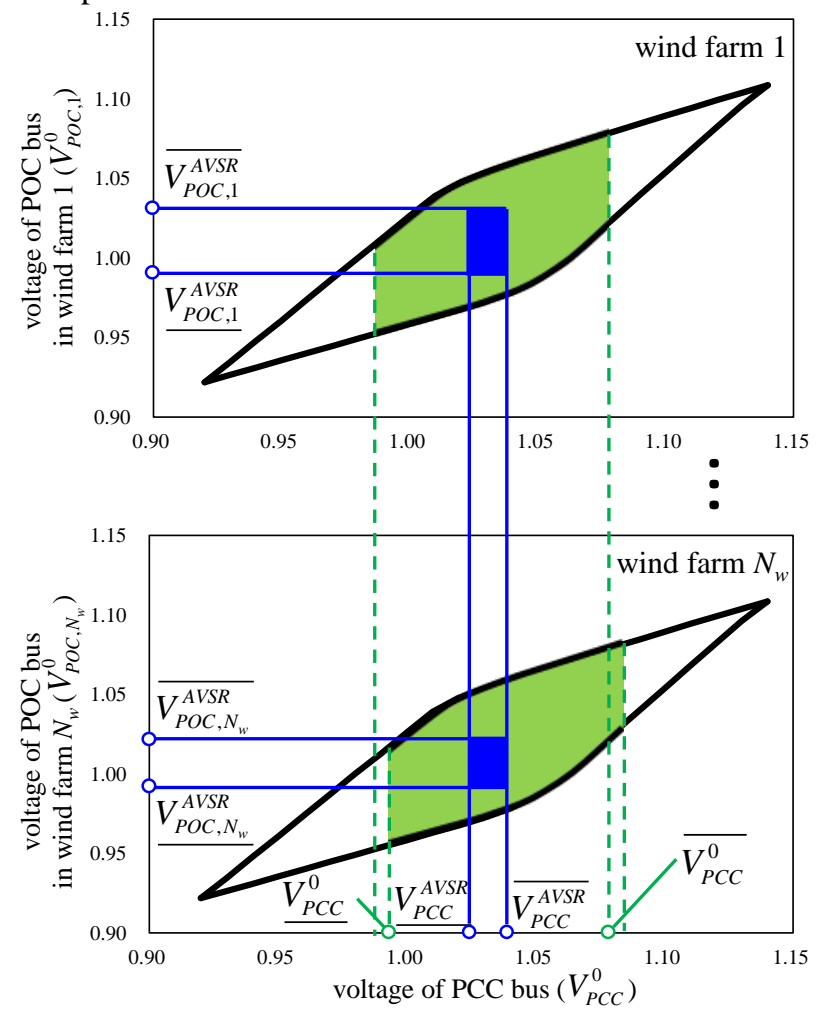

Fig. 5. AVSR for multiple wind farms

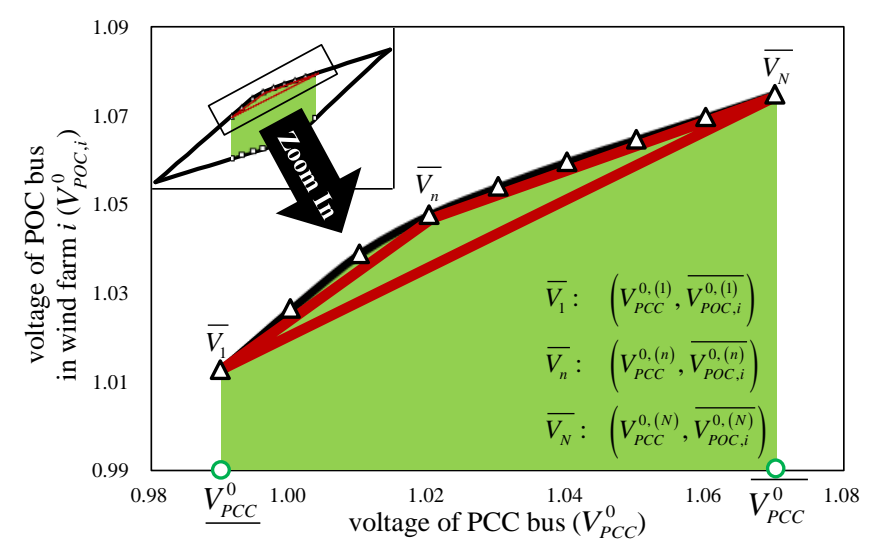

Fig. 6. Linearization of VFR's upper bound for wind farm $i$

To compute the AVSR, the upper bounds and lower bounds of all wind farms' practical VFRs (green regions) are first linearized by two lines respectively for practical application. As shown in Fig. 6, there are $N$ operational points on VFR's upper bound (from $\overline{V_{1}}$ to $\overline{V_{N}}$ ). (the upper-left small subfigure in Fig. 6 is the voltage feasible region, i.e. Fig. 4) The upper bound is better approximately linearized with a larger square of the triangle $\overline{V_{1} V_{n} V_{N}}$. It is assumed that $n=n^{*}$ is the optimal of total $N$ points (2-a), then the two linearized constraints of the upper bound (line $\overline{V_{1} V_{n}}$ and $\overline{V_{n} V_{N}}$ ) can be expressed as (2-c). Similar to the upper bound, this method can be also applied to the linearization of the lower bound. There are also $N$ operational points on VFR's lower bound (from $\underline{V_{1}}$ to $\underline{V_{N}}$ ). The square of the triangle $\underline{V_{1} V_{m} V_{N}}$ reaches the maximum when $m=m^{*}$ (2-b), then the two linearized constraints of the lower bound (line $\underline{V_{1} V_{m}}$ and $\underline{V_{m} V_{N}}$ ) can be expressed as (2-d). ( $\underline{V_{m}}$ is on the lower bound)

$$
\begin{aligned}
& n^{*}=\arg \max _{n=1,2, \ldots N} S_{\Delta \overline{V_{1}} \overline{V_{n}} \overline{V_{N}}} \\
& m^{*}=\arg \max _{m=1,2, \ldots N} S_{\Delta \underline{V_{1}} \underline{V_{m}} \underline{V_{N}}} \\
& V_{P O C, i}^{0}-\overline{V_{P O C, i}^{0,(1)}} \leq \frac{\overline{V_{P O C, i}^{0,\left(n^{*}\right)}}-\overline{V_{P O C, i}^{0,(1)}}}{V_{P C C}^{0,\left(n^{*}\right)}-V_{P C C}^{0,(1)}}\left(V_{P C C}^{0}-V_{P C C}^{0,(1)}\right) \\
& V_{P O C, i}^{0}-\overline{V_{P O C, i}^{0,(N)}} \leq \frac{\overline{V_{P O C, i}^{0,\left(n^{*}\right)}}-\overline{V_{P O C, i}^{0,(N)}}}{V_{P C C}^{0,\left(n^{*}\right)}-V_{P C C}^{0,(N)}}\left(V_{P C C}^{0}-V_{P C C}^{0,(N)}\right)
\end{aligned}
$$

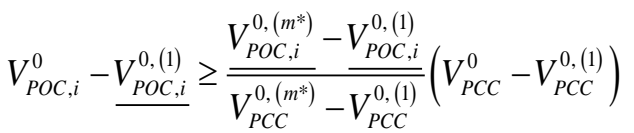

$$
\begin{aligned}
& V_{P O C, i}^{0}-\underline{V_{P O C, i}^{0,(N)}} \geq \frac{V_{P O C, i}^{0,\left(m^{*}\right)}-V_{P O C, i}^{0,(N)}}{\overline{V_{P C C}^{0,\left(m^{*}\right)}-V_{P C C}^{0,(N)}}}\left(V_{P C C}^{0}-V_{P C C}^{0,(N)}\right)
\end{aligned}
$$

Here, $\underline{V_{P C C}^{0}}$ and $\overline{V_{P C C}^{0}}$ are set to the maximum and minimum value among all wind farms (2-e), as shown in Fig. 5.

$$
\underline{V_{P C C}^{0}} \leq V_{P C C}^{0} \leq \overline{V_{P C C}^{0}}
$$

For convenience, (2-f) is used instead of the VFR's boundary constraints (2-c) (2-e), expressing that the operation point $\left(V_{P C C}^{0}, V_{P O C, i}^{0}\right)$ is within the VFR of wind farm $i$.

$$
\boldsymbol{f}_{i}\left(V_{P C C}^{0}, V_{P O C, i}^{0}\right) \leq 0
$$

(2) completes the linearization of the VFR, which is used in SCOPF (3).

The AVSR for multiple wind farms can also be obtained by using stepwise search method. For each step at the given $V_{P C C}^{0}$, the corresponding operation range $\left[\underline{V_{P O C, i}^{0}}, \overline{V_{P O C, i}^{0}}\right]$ for $V_{P O C, i}^{0}$ in the individual wind farm $i$ can be computed by (1) above. But in multiple wind farms, the normal operation range for $V_{P O C, i}^{0}$, noted as $\left[V_{P O C, i(1)}^{0}, V_{P O C, i(2)}^{0}\right]$, is a subrange of $\left[V_{P O C, i}^{0}, \overline{V_{P O C, i}^{0}}\right]$ (as shown in Fig. 7) due to the following two reasons:

1) The operation range $\left[V_{P O C, i}^{0}, \overline{V_{P O C, i}^{0}}\right]$ of the individual wind farm $i$ is computed based on the assumption that the control center has enough control capability to control $V_{P C C}^{0}$ at the given $V_{P C C}^{0, S P}(1-\mathrm{g})$. But in multiple wind farms, the control center may not have enough control capability to control $V_{P C C}^{0}$ at $V_{P C C}^{0, S P}$ while wind farms decoupled control their $V_{P O C, i}^{0}$ at any value within $\left[V_{P O C, i}^{0}, \overline{V_{P O C, i}^{0}}\right]$. For example, the voltage operation point 
$\left.\overline{\left(V_{P O C, 1}^{0}\right.}, \overline{V_{P O C, 2}^{0}}, \ldots, \overline{V_{P O C, N w}^{0}}, V_{P C C}^{0, S P}\right)$ may not exist.

2) $\left[V_{P O C, i(1)}^{0}, V_{P O C, i(2)}^{0}\right]$ should both guarantee all WTGs' normal operation not only in normal conditions but also under any $N-1$ contingency.

$$
\begin{aligned}
& \max _{\boldsymbol{\Delta} \boldsymbol{Q}_{w}^{0}, \boldsymbol{\Delta} \mathbf{Q}_{g}^{0}, \boldsymbol{\Delta} \boldsymbol{Q}_{s v c}^{s}} f_{1}-\lambda f_{2} \\
& f_{1}=\sum_{i=1}^{N_{w}} \alpha_{i} \frac{V_{P O C, i(2)}^{0}-V_{P O C, i(1)}^{0}}{\overline{V_{P O C, i}^{0}}-V_{P O C, i}^{0}} \\
& f_{2}=\sum_{k=1}^{2} \sum_{s=1}^{N_{s}} \sum_{i=1}^{N_{w}}\left\|V_{P O C, i(k)}^{s}-V_{P O C, i(k)}^{0}\right\| \\
& \text { s.t. } \Delta Q_{g, m(k)}^{0} \leq \Delta Q_{g, m(k)}^{0} \leq \overline{\Delta Q_{g, m(k)}^{0}} \\
& \underline{Q_{i}^{0}} \leq Q_{i}^{0, \text { current }}+\Delta Q_{i(k)}^{0} \leq \overline{Q_{i}^{0}} \\
& \underline{V_{P O C, i}^{0}} \leq V_{P O C, i(k)}^{0} \leq \overline{V_{P O C, i}^{0}} \\
& V_{P O C, i(k)}^{0}=V_{P O C, i}^{0, \text { current }}+\sum_{m=1}^{N_{g}} S_{p w, m i}^{0, q v} \Delta Q_{g, m(k)}^{0}+\sum_{j=1}^{N_{w}} S_{w w, j i}^{0, q v} \Delta Q_{j(k)}^{0} \\
& V_{P C C}^{0, S P}=V_{P C C}^{0, \text { current }}+\sum_{m=1}^{N_{g}} S_{p p, m}^{0, q v} \Delta Q_{g, m(k)}^{0}+\sum_{j=1}^{N_{w}} S_{w p, j}^{0, q v} \Delta Q_{j(k)}^{0} \\
& \underline{Q_{s v c, i}^{s}} \leq \Delta Q_{s v c, i(k)}^{s} \leq \overline{Q_{s v c, i}^{s}} \\
& \boldsymbol{f}_{i}\left(V_{P C C(k)}^{s}, V_{P O C, i(k)}^{s}\right) \leq 0 \\
& V_{P O C, i(k)}^{s}=V_{P O C, i}^{s, c u r r e n t}+\sum_{j=1}^{N_{w}} S_{w w, j i}^{s, q v} \Delta Q_{s v c, j(k)}^{s} \\
& V_{P C C(k)}^{s}=V_{P C C}^{s, \text { current }}+\sum_{j=1}^{N_{w}} S_{w p, j}^{s, q v} \Delta Q_{s v c, j(k)}^{s} \\
& i=1,2, \ldots, N_{w}, \quad m=1,2, \ldots, N_{g} \\
& s=1,2, \ldots, N_{s}, \quad k=1,2
\end{aligned}
$$

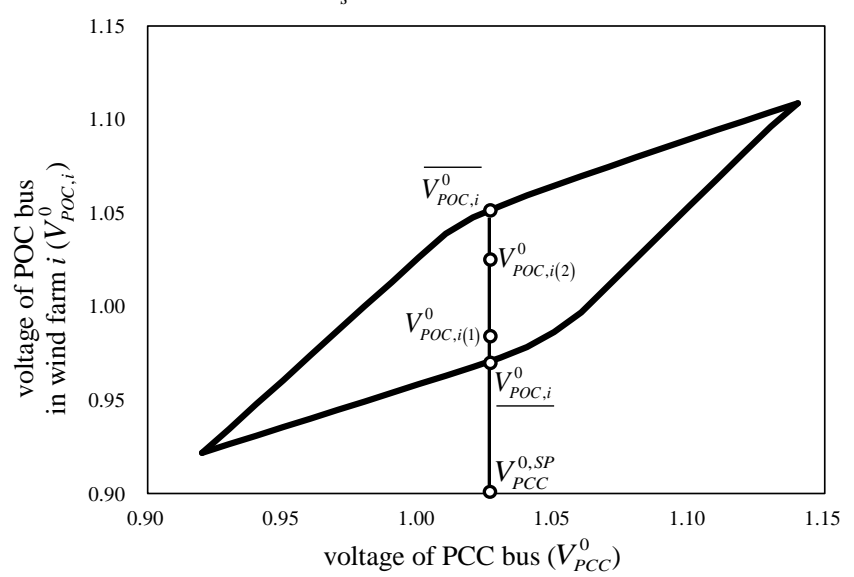

Fig. 7. Voltage operation range of wind farm $i$ in the individual wind farm and in multiple wind farms for each given $V_{P C C}^{0, S P}$

Therefore, for each given $V_{P C C}^{0, S P}(3-\mathrm{e})$, the control center aims to seek the largest voltage operation range $\left(f_{1}\right.$ in (3-a)) for the POC buses of all wind farms. Under normal conditions, the conventional power plants use reactive power control capabilities (3-b) to control $V_{P C C}^{0}$ to the given value $V_{P C C}^{0, S P}$ (3-e), while wind farms use reactive power control capabilities (3-c) to maintain $V_{P O C, i}^{0}$ within $\left[V_{P O C, i}^{0}, \overline{V_{P O C, i}^{0}}\right](3-\mathrm{d})$. Wind farms cannot operate normally if $\overline{(3-\mathrm{d})}$ is violated in the normal conditions. When a $N-1$ contingency happens, the fast-response compensators, such as SVCs, are regulated (3-f) in order to maintain POC buses' voltage as much as they can $\left(f_{2}\right.$ in (3-a)), which also guarantees that the operation points are within the VFR (3-g) after $N-1$ contingency, the violation of constraints (3-g) may result in a cascading trip. For a wind pool area, all individual wind farms' trip faults should be considered, otherwise any trip fault is likely to cause a cascading trip fault triggered by the first trip fault. Here $S$ is the sensitivity coefficient, e.g. $S_{w p, j}^{0, q v}$ denotes the sensitivity of PCC's $(p)$ voltage $(v)$ to wind farm $(w) j$ 's reactive power $(q)$ under normal conditions (superscript 0).

It should be noted:

1) $\alpha_{i}$ is the weight coefficient for each wind farm. The weight coefficient for wind farms with a larger capacity of reactive power compensators is greater.

2) The weight coefficient $\lambda$ and $\alpha_{i}$ in multiple objective function (3-a) must satisfy $\lambda \gg \alpha_{i}, i=1,2, \ldots, N_{w}$.

For each step at the given $V_{P C C}^{0, S P},\left[V_{P O C, i(1)}^{0}, V_{P O C, i(2)}^{0}\right]$ solved by (3) can guarantee decoupling and security:

(A) Decoupling: Wind farm $i$ can realize decoupled control $V_{P O C, i}^{0}$ at any value within $\left[V_{P O C, i(1)}^{0}, V_{P O C, i(2)}^{0}\right]$ without considering other wind farms. (Proved below)

(B) Security: Normal operation for all wind farms under normal conditions and under any $N-1$ contingency.

Proof of (A): Firstly, let $\Delta V_{\mathrm{g}(k)}^{0}$ denote the total voltage regulation of conventional generators at the current operation state

$$
\Delta V_{g(k)}^{0}=\sum_{m=1}^{N_{g}} S_{p w, m i}^{0, q v} \Delta Q_{g, m(k)}^{0}
$$

where $\Delta Q_{g, m(k)}^{0}$ is constrained by (3-b), thus $\Delta V_{\mathrm{g}(k)}^{0}$ is constrained $\Delta V_{\mathrm{g}}^{0} \leq \Delta V_{\mathrm{g}(k)}^{0} \leq \overline{\Delta V_{\mathrm{g}}^{0}}$. It is assumed $\left(V_{P C C}^{0, S P}, \Delta V_{\mathrm{g}(1)}^{0\left(^{*}\right)}\right.$, $\left.V_{P O C, 1(1)}^{O(*)}, \ldots, V_{P O C, N_{w}(1)}^{0\left(^{*}\right)}, \Delta V_{\mathrm{g}(2)}^{0(*)}, V_{P O C, 1(2)}^{0\left(^{*}\right)}, \ldots, V_{P O C, N_{w}(2)}^{0\left(^{*}\right)}\right)$ is optimal of OPF (3).

When the voltage of PCC bus $V_{P C C}^{0}$ is fixed at $V_{P C C}^{0, S P}$, for wind farm $i$, the voltage of its POC bus $V_{P O C, i}^{0}$ is strictly increasing in its total reactive power regulation $\Delta Q_{i}^{0}$, the strictly increasing function is defined as

$$
V_{P O C, i}^{0}=\left.F_{i}\right|_{V_{P C C}^{0}=V_{P C C}^{0, S P}}\left(\Delta Q_{i}^{0}\right)
$$

where $\Delta Q_{i}^{0}=Q_{i}^{0}-Q_{i}^{0, \text { current }}$. For wind farm $i\left(i=1,2, \ldots, N_{w}\right)$, $\forall V_{P O C, i}^{0} \in\left[V_{P O C, i(1)}^{O(*)}, V_{P O C, i(2)}^{0\left(^{*}\right)}\right]$, because the inverse function

$$
\Delta Q_{i}^{0}=\left.F_{i}^{-1}\right|_{V_{P C C}^{0}=V_{P C C}^{0, S P}}\left(V_{P O C, i}^{0}\right)
$$

is also strictly increasing, as such,

$$
\begin{aligned}
\underline{Q_{i}^{0}}-Q_{i}^{0, \text { current }} & \leq \Delta Q_{i(1)}^{0(*)} \leq \Delta Q_{i}^{0} \leq \Delta Q_{i(2)}^{0(*)} \leq \overline{Q_{i}^{0}}-Q_{i}^{0, \text { current }} \\
& \Rightarrow \underline{Q_{i}^{0}} \leq \Delta Q_{i}^{0}+Q_{i}^{0, \text { current }} \leq \overline{Q_{i}^{0}}
\end{aligned}
$$

And the sensitivity coefficient $S_{w p, j}^{0, q v}$ is always positive, then 


$$
\begin{aligned}
& V_{P C C}^{0, S P}-V_{P C C}^{0, \text { current }}-\overline{\Delta V_{g}^{0}} \leq V_{P C C}^{0, S P}-V_{P C C}^{0, \text { current }}-\Delta V_{g(1)}^{0(*)} \\
& =\sum_{i=1}^{N_{w}} S_{w p, i}^{0, q v} \Delta Q_{i(1)}^{0(*)} \leq \sum_{i=1}^{N_{w}} S_{w p, i}^{0, q v} \Delta Q_{i}^{0}=\sum_{i=1}^{N_{w}} S_{w p, i}^{0, q v} \Delta Q_{i(2)}^{0(*)}= \\
& V_{P C C}^{0, S P}-V_{P C C}^{0, \text { current }}-\Delta V_{g(2)}^{0(*)} \leq V_{P C C}^{0, S P}-V_{P C C}^{0, \text { current }}-\underline{\Delta V_{g}^{0}} \\
& \Rightarrow \quad \underline{\Delta V_{g}^{0}} \leq V_{P C C}^{0, S P}-V_{P C C}^{0, \text { current }}-\sum_{i=1}^{N_{w}} S_{w p, i}^{0, q v} \Delta Q_{i}^{0} \leq \overline{\Delta V_{g}^{0}}
\end{aligned}
$$

Due to the fact that $\Delta Q_{g, m}^{0}$ are continuous control variables, $\Delta Q_{g, m}^{0}$ can be obtained such that

$$
\left\{\begin{array}{l}
V_{P C C}^{0, S P}=V_{P C C}^{0, \text { current }}+\Delta V_{g}^{0}+\sum_{i=1}^{N_{w}} S_{w p, i}^{0, q v} \Delta Q_{i}^{0} \\
\frac{\Delta V_{g}^{0}}{\underline{\Delta Q_{g, m}^{0}}} \leq \Delta V_{g}^{0}=\Delta \sum_{m=1}^{N_{g}} \leq \overline{\Delta Q_{g, m}^{0}} S_{p, m i}^{0, q v} \Delta Q_{g, m}^{0} \leq \overline{\Delta V_{g}^{0}}
\end{array}\right.
$$

Thus $\forall V_{P O C, i}^{0} \in\left[V_{P O C, i(1)}^{0\left(^{* *}\right)}, V_{P O C, i(2)}^{\left.00^{*}\right)}\right]$, a corresponding $\Delta Q_{i}^{0}$ can always be found for wind farm $i, \Delta Q_{g, m}^{0}$ for conventional generators which satisfy all constraints at the fixed value $V_{P C C}^{0, S P}$ of PCC bus, i.e., the control center can use conventional generators' control capabilities to control the PCC bus's voltage at $V_{P C C}^{0, S P}$ when each wind farm decoupled control their POC buses' voltage within $\left[V_{P O C, i(1)}^{0\left(^{*}\right)}, V_{P O C, i(2)}^{0\left(^{*}\right)}\right]$.

This completes the proof.

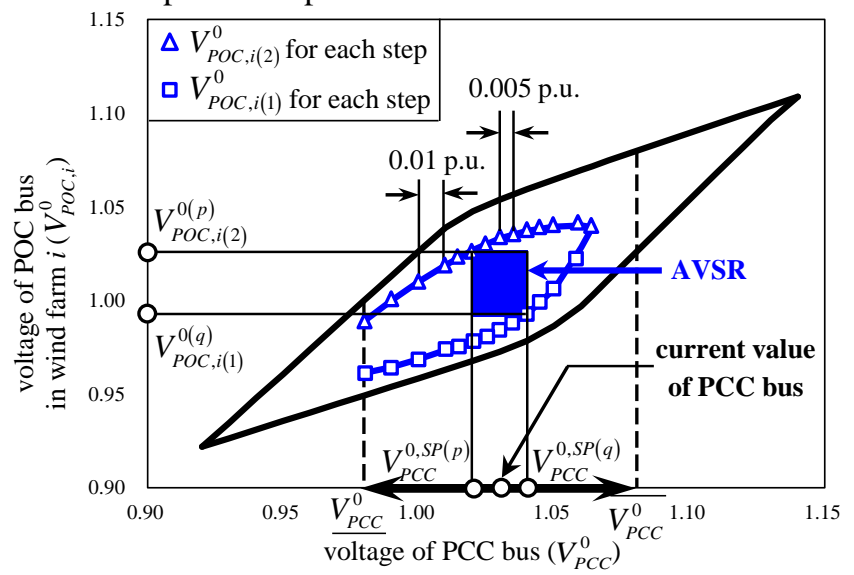

Fig. 8. AVSR for wind farm $i$

Therefore, the stepwise search method can be used to obtain the AVSR. Similar to the VFR above, from the wind farm side, let $V_{P C C}^{0}$ increase and decrease stepwise respectively from current value, the corresponding $V_{P O C, i(1)}^{0}$ and $V_{P O C, i(2)}^{0}$ is computed for each given $V_{P C C}^{0, S P}$ by solving (3), then combine these solution points together to acquire the voltage security region curves, as shown in Fig. 8. Considering both accuracy and efficiency, the first several steps in the computation are set to 0.005 p.u. while other steps are set to 0.01 p.u..

Apparently, there are many different rectangles within the blue curves in Fig. 8. It is therefore important to seek the largest AVSR for the wind pool area. To find the largest AVSR, $V_{P O C, i(1)}^{0}$ and $V_{P O C, i(2)}^{0}$ are first normalized for each given $V_{P C C}^{0, S P}$ in Fig. 7 using (4).

$$
V_{P O C, i(k)}^{0}=\frac{V_{P O C, i(k)}^{0}-\underline{V_{P O C, i}^{0}}}{\overline{V_{P O C, i}^{0}}-\underline{V_{P O C, i}^{0}}}, \quad k=1,2
$$

Then the square of AVSR can be computed using (5), the superscript $p$ and $q$ represent step $p$ and $q$, as shown in Fig. 8 . The AVSR can be obtained from the maximum $S(p, q)$ expressed in (6), where the stars follow $p$ or $q$ represent the maximum solution of all steps. Another point should be noted is the requirement of accuracy. In addition to decreasing the search step in Fig. 4 and Fig. 8, linear interpolation can be used between two adjacent steps in Fig. 8 to get more data points in order to obtain a larger AVSR.

$$
\begin{aligned}
& S(p, q)=\left(V_{P C C}^{0, S P(q)}-V_{P C C}^{0, S P(p)}\right) \cdot \sum_{i=1}^{N_{w}} \alpha_{i}\left(V_{P O C, i(2)}^{0(p)}-V_{P O C, i(1)}^{0(q)}\right) \\
& \underline{V_{P O C, i}^{A V S R}}=V_{P O C, i(1)}^{0\left(q^{*}\right)}, \quad \overline{V_{P O C, i}^{A V S R}}=V_{P O C, i(2)}^{0\left(p^{*}\right)} \\
& \underline{V_{P C C}^{A V S R}}=V_{P C C}^{0, S P\left(p^{*}\right)}, \quad \overline{V_{P C C}^{A V S R}}=V_{P C C}^{0, S P\left(q^{*}\right)}
\end{aligned}
$$

\section{The computation process of AVSR in a wind pool area}

The process of acquiring AVSR can be divided into six steps, as shown in table I, the information exchange and six computation steps between system-side and wind-farm-side are shown in Fig. 9.

TABLE I

FLOW CHART TO COMPUTE AVSR

$$
\text { FLOW CHART }
$$

Step 1 All the individual wind farms use stepwise search method for each given $V_{P C C}^{0, S P}$ to compute the corresponding $\left[V_{P O C, i}^{0}, \overline{V_{P O C, i}^{0}}\right]$ by (1).

Step 2 Each individual wind farm sends the data set $\left\{\boldsymbol{V}_{P C C}^{0, S P}, \underline{\boldsymbol{V}_{P O C, i}^{0}}, \overline{\boldsymbol{V}_{P O C, i}^{0}}, \underline{\boldsymbol{Q}_{i}^{0}}, \overline{\boldsymbol{Q}_{i}^{0}}\right\}$ to control center.

Step 3 The control center uses (2) to linearize the upper bounds and lower bounds of all wind farms.

Step 4 The control center uses stepwise search method for each given $V_{P C C}^{0, S P}$ to compute the corresponding $\left[V_{P O C, i(1)}^{0}, V_{P O C, i(2)}^{0}\right]$ by (3).

Step 5 Normalize each $V_{P O C, i(k)}^{0}$ using (4) and obtain the largest AVSR using (5). The result of AVSR is expressed as (6).

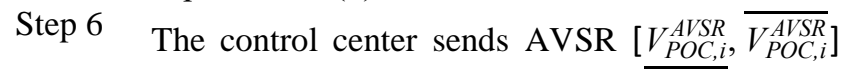
back to each wind farm for voltage control. The control center should control the voltage of PCC bus within $\left[V_{P C C}^{A V S R}, \overline{V_{P C C}^{A V S R}}\right]$. 


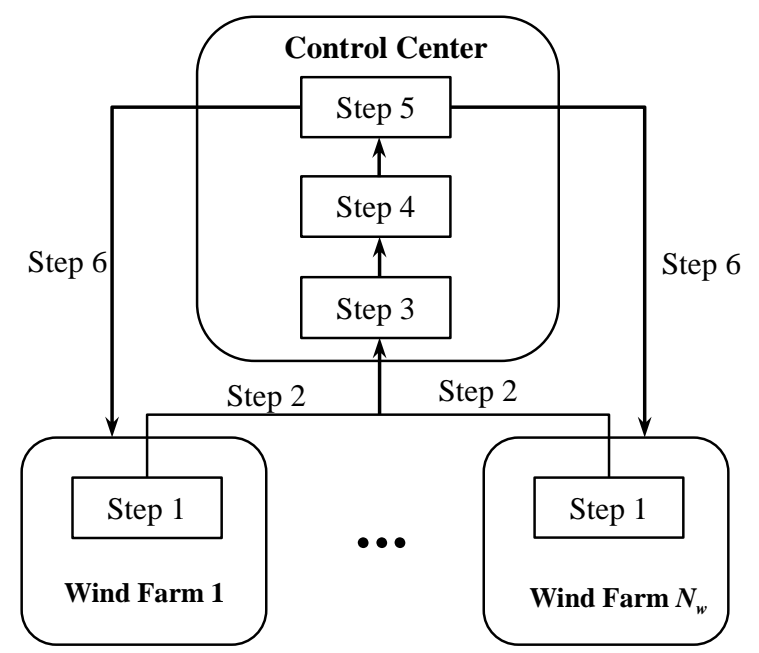

Fig. 9. Information exchange and six computation steps of AVSR between system-side and wind-farm-side

For more clear illustration and understanding, we put all regions and curves into Fig. 10, which also shows the corres ponding step in table I to compute or get these regions and curves.

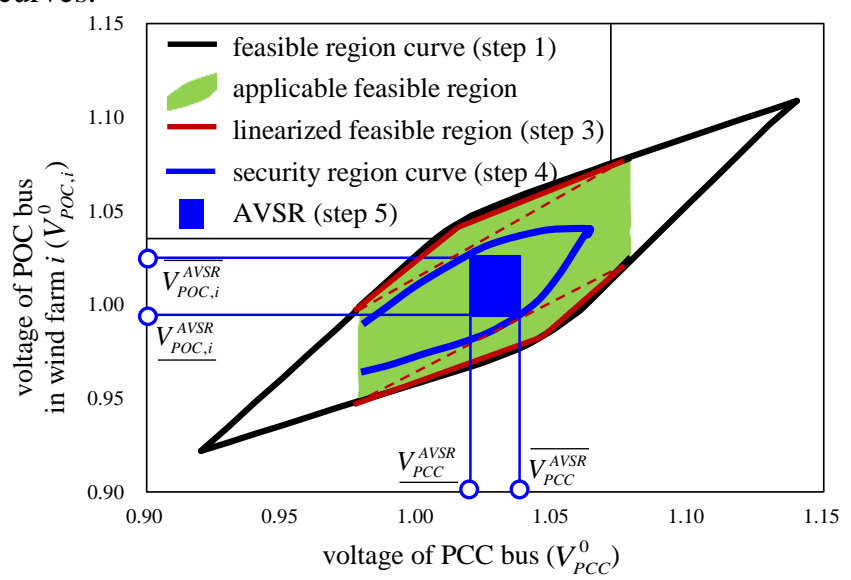

Fig. 10. Illustration of different kinds of regions and curves

\section{CASE STUdies}

A. A simple system with two wind farms

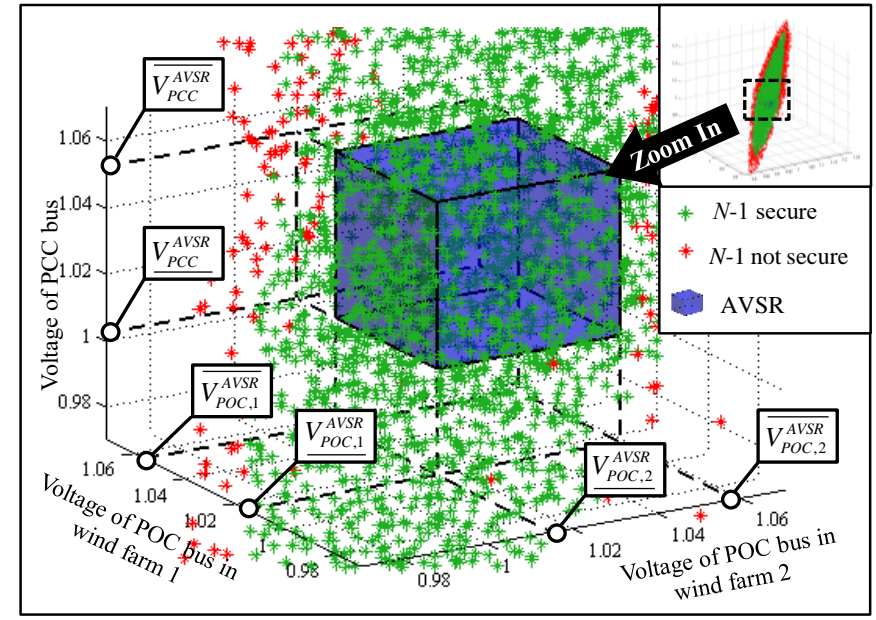

Fig. 11. AVSR for two wind farms and PCC bus
A simple system with two wind farms was studied to verify the proposed method using Monte Carlo simulation. First, the Monte Carlo simulation method was used to generate 10,000 simulation points that guarantee normal operation under $\mathrm{N}-0$ conditions, thereafter all $N$-1 contingencies based on each operation point were computed. The " $N-1$ secure points" were plotted green while " $N$-1 not secure points" were plotted red in Fig. 11. Then, the AVSR (blue cube) was computed for this simple system and plotted in Fig. 11. The simulation results demonstrate three characteristics of AVSR:

1) Security: All operational points in the AVSR were green points ("N-1 secure points").

2) Accuracy: $N-1$ security cannot be guaranteed if operational points are located outside the blue cube in Fig. 11. Some red points ("N-1 not secure points") exist outside but near to the cube boundary, which shows that the boundary of computed AVSR is near to the real boundary of security region.

3) Autonomous (Decoupling): The operation points fill all space of the blue cube, i.e. wind farm 1 and wind farm 2 can controls its own POC bus's voltage to any value within $\left[V_{P O C, 1}^{A V S R}\right.$, $\left.\overline{V_{P O C, 1}^{A V S R}}\right]$ and $\left[V_{P O C, 2}^{A V S R}, \overline{V_{P O C, 2}^{A V S R}}\right]$ by itself as long as the PCC bus controls its voltage within $\left[V_{P C C}^{A V S R}, \overline{V_{P C C}^{A V S R}}\right]$, without considering voltage operation details of the other wind farm and the PCC bus.

Therefore, the AVSR proposed in this paper can be used for voltage control in a decoupled way.

\section{B. A real system with eight wind farms in Northern China}

A real system with eight wind farms comprising the ZB Wind Power Base in North China, as Fig. 2 shows, was studied to verify the effectiveness of the AVSRs proposed in this study. Firstly, the time and frequency computed once for wind farms are recorded in table II. Due to the higher linearity DistFlow model in wind farms and only once necessary information exchange, the computation does not take a lot of time.

Fig. 12 shows the voltage deviation in the $\mathrm{ZB}$ wind power base after different $N-1$ contingencies. There are eight black lines, which represent eight different contingencies for eight wind farms. If eight wind farms and the PCC substation operate within their own AVSR, then after an $N-1$ contingency, even if the voltages of other wind farms and the PCC substation increase, they will not violate the voltages' upper and lower bounds of normal operation.

TABLE II

COMPUTATION TIME AND FREQUENCY FOR Wind FARMS

\begin{tabular}{ccccc}
\hline \hline $\begin{array}{c}\text { Computa- } \\
\text { tion Place }\end{array}$ & $\begin{array}{c}\text { OPF/ } \\
\text { SCOPF }\end{array}$ & $\begin{array}{c}\text { Computing } \\
\text { Frequency }\end{array}$ & $\begin{array}{c}\text { Computing } \\
\text { Time }\end{array}$ & $\begin{array}{c}\text { Total } \\
\text { Time }\end{array}$ \\
\hline \multirow{5}{*}{ Each } & CNG & 24 & $7.0456 \mathrm{~s}$ & \\
Individual & GT & 22 & $7.1784 \mathrm{~s}$ & \\
Wind Farm & WLS & 26 & $6.9729 \mathrm{~s}$ & \\
& GH & 22 & $7.6053 \mathrm{~s}$ & $7.6053 \mathrm{~s}$ \\
& WHP & 24 & $7.1245 \mathrm{~s}$ & \\
Control & QSY & 24 & $7.3876 \mathrm{~s}$ & \\
Center & Security & 13 & $8.7761 \mathrm{~s}$ & $8.7761 \mathrm{~s}$ \\
Other computations (include boundary linearization, & $0.0245 \mathrm{~s}$
\end{tabular}


normalization by (4), acquire the largest AVSR by (5)) Total Computation Time

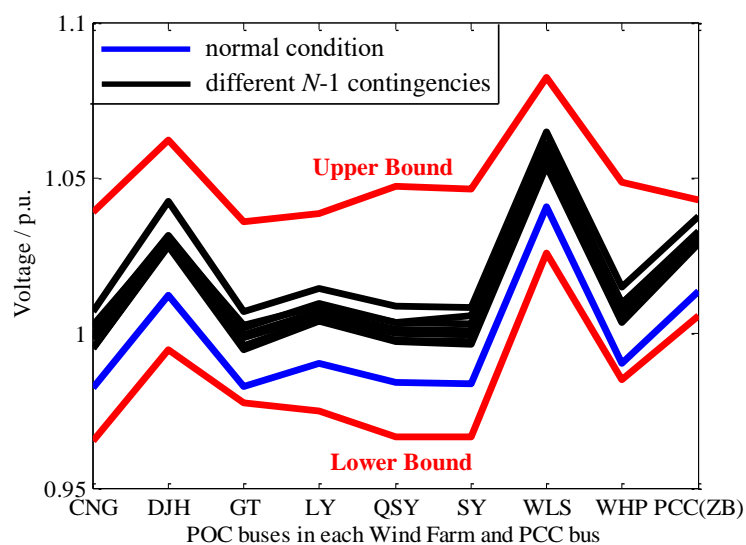

Fig. 12. Voltage of wind farms and the PCC bus within an AVSR after $N-1$ contingencies

Fig. 13 shows the voltage of each WTG in an individual wind farm, in which each white star represents a WTG. If a wind farm operates within its AVSR, as shown in Fig. 13 (a.1) and (a.2), then the WTGs operate at a lower voltage level before a trip fault. After other wind farms' trip fault, the voltage of all WTGs will increase, but they will not exceed the upper bound (1.10 p.u.). However, the situation is different if the wind farm operates without the AVSR, which is shown in Fig. 13 (b.1) and (b.2). Before the trip fault, although each WTG can operate normally, WTGs in the white cycle operate at a higher voltage level and are close to the upper bounds (1.10 p.u.). After other wind farms' trip fault, all WTGs' voltage increases and some (WTGs in the white cycle) exceed their upper bounds, leading to cascading trip faults.

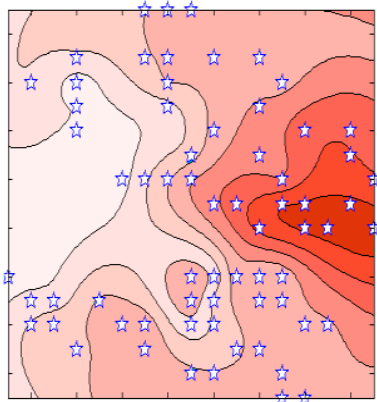

(a.1) Before trip fault

(a) within AVSR

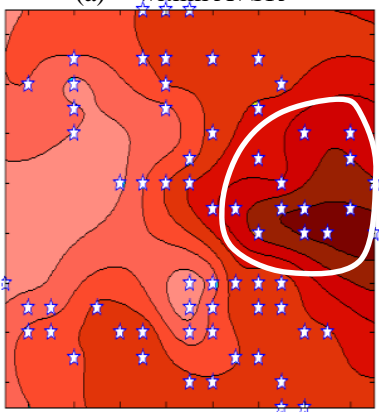

(b.1) Before trip fault

(b) without AVSR

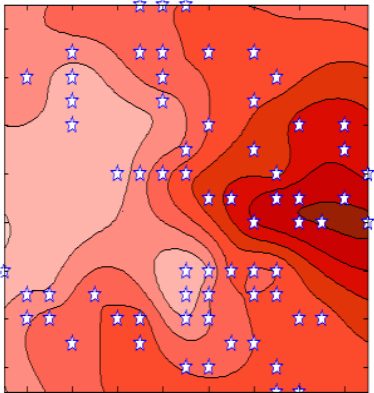

(a.2) After trip fault

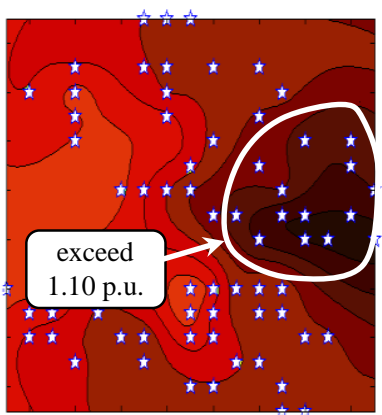

(b.2) After trip fault
Fig. 13. Voltage magnitude of wind units in a wind farm within or without the AVSR after an $N-1$ contingency

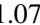

\section{Comparison with other works}

Here we compare the proposed AVSR with recent relevant researches from eight aspects in table III. By comparison, the advantages of the proposed method can be summarized as follows

1) Voltage security regions (VSR) are decoupled or not: For each wind farm, the proposed AVSR $\left[V_{P O C, i}^{A V S R}, \overline{V_{P O C, i}^{A V S R}}\right]$ can be used for decoupled voltage control, while other works compute the VSR $\left[V_{i}^{0}, \overline{V_{i}^{0}}\right]$ which cannot be used for decoupled voltage control. (As shown in Fig. 14)

2) The proposed AVSR also provided decoupled security voltage ranges $\left[V_{P C C}^{A V S R}, \overline{V_{P C C}^{A V S R}}\right]$ for PCC substation, but other works didn't.

3) In each wind farm, the power flow model (in DistFlow format) is used. Thus the result of the proposed method will be more accurate.

4) The stepwise search method proposed in this paper does not require iteration, and the necessary constraint information is exchanged only once between the control center and each wind farm, resulting in less necessary computation time. Here we use 100 different power flow interfaces to compute VSR of the real system (as Fig. 2 shows) using different methods. The average total computation time was recorded in table III and the proposed method observes faster computations than other works.

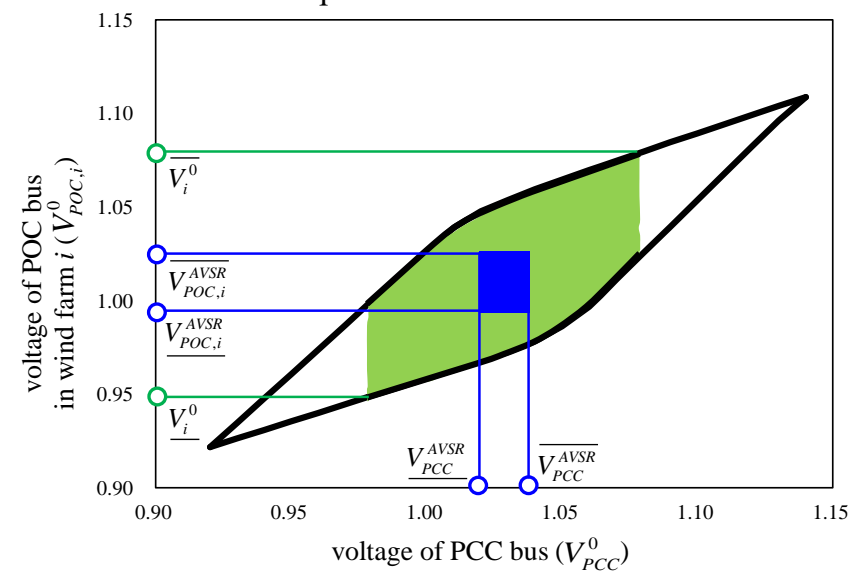

Fig. 14. Comparison between AVSR proposed in this paper and VSR in relevant works

TABLE III

COMPARISON AMONG DIFFERENT METHODS

\begin{tabular}{|c|c|c|c|c|}
\hline \multicolumn{2}{|c|}{ Comparison } & This paper & [22] & [20], [21] \\
\hline \multicolumn{2}{|c|}{$\begin{array}{c}\text { Results of security region } \\
\text { for wind farms }\end{array}$} & {$\left[V_{P O C, i}^{A V S R}, \overline{V_{P O C, i}^{A V S R}}\right]$} & {$\left[\underline{V_{i}^{0}}, \overline{V_{i}^{0}}\right]$} & {$\left[\underline{V_{i}^{0}}, \overline{V_{i}^{0}}\right]$} \\
\hline \multicolumn{2}{|c|}{$\begin{array}{l}\text { Wind farms can decoupled } \\
\text { control their voltages? }\end{array}$} & Yes & No & No \\
\hline \multicolumn{2}{|c|}{$\begin{array}{l}\text { Provide decoupled } \\
\text { security voltage ranges for } \\
\text { PCC substation? }\end{array}$} & {$\left[\underline{V_{P C C}^{A V S R}}, \overline{V_{P C C}^{A V S R}}\right]$} & No & No \\
\hline \multicolumn{2}{|c|}{ Main computation method } & Stepwise search & Iteration & $\begin{array}{l}\text { Approximate } \\
\text { boundary }\end{array}$ \\
\hline \multirow{2}{*}{$\begin{array}{l}\text { Model and } \\
\text { Accuracy }\end{array}$} & $\begin{array}{l}\text { Wind } \\
\text { Farms }\end{array}$ & $\begin{array}{c}\text { Power flow } \\
\text { model in } \\
\text { DistFlow format }\end{array}$ & $\begin{array}{l}\text { Sensitive } \\
\text { model }\end{array}$ & $\begin{array}{l}\text { Sensitive } \\
\text { model }\end{array}$ \\
\hline & $\begin{array}{l}\text { Control } \\
\text { Center }\end{array}$ & \multicolumn{3}{|c|}{ Sensitive model } \\
\hline
\end{tabular}




\begin{tabular}{c|ccc}
\cline { 2 - 4 } Accuracy & better & average & average \\
\hline $\begin{array}{c}\text { Average total computation } \\
\text { time for 100 computations }\end{array}$ & $15.25 \mathrm{~s}$ & $16.11 \mathrm{~s}$ & $15.48 \mathrm{~s}$ \\
\hline $\begin{array}{c}\text { Necessary information } \\
\text { exchange during computa- } \\
\text { tions }\end{array}$ & Only once & $\begin{array}{c}\text { Require } \\
\text { iterations }\end{array}$ & Only once \\
\hline $\begin{array}{c}\text { Consider randomness of } \\
\text { wind power? }\end{array}$ & No & Yes & No \\
\hline \hline
\end{tabular}

\section{CONCLUSIONS}

Due to the complexity of voltage security regions and the difficulty of computation, this paper proposes the AVSR and a stepwise search method for its computation. At each wind farm, an AVSR is designed to guarantee the normal operation of each WTG, while in the control center, each region is designed in order to guarantee normal operation both under normal conditions and after $\mathrm{N}-1$ contingencies. If the control center controls PCC bus within its AVSR and all the wind farms' POC buses operate in their own AVSR, each wind farm can realize decoupled control of all the WTGs within normal operation ranges both under normal conditions and under any $N-1$ contingency without considering the operation details of other wind farms.

Compared with the previous methods, the results are more accurate because the power flow model is used instead of a traditional sensitive model in each wind farm. It will not take a lot of computation time because this method does not require iteration and uses power flow model with the DistFlow format in each wind farm. Case studies with s simple system and a real system verify the accuracy and effectiveness of the method, and good performance using a Monte Carlo simulation.

With increased wind power penetration, AVSRs may not exist. Thus, how to curtail wind power and acquire maximum security regions will be studied in a future study. Indeed, it's also important to deal with the randomness of active power due to the forecast errors. Compared with the AVSR proposed in this paper, the AVSR robust to active wind power randomness is much more complicated and the computation must take more time. Based on the proposed AVSR using the specific current active power interfaces in this paper, the AVSR robust to active wind power randomness will be studied in the future works. As we all known, systems dynamics play an important role in wind security. How dynamics influences the AVSR (e.g. the optimal allocation of SVC/SVGs) in wind farms, which is more complicated, which will be studied in future work.

\section{REFERENCES}

[1] A. A. Tamimi, A. Pahwa and S. Starrett, "Method for assessing system impact of increasing wind farm sizes above their maximum limits," in Power and Energy Society General Meeting, 2011 IEEE, San Diego, CA, 2011, pp. 1-8.

[2] A.A. Tamimi, A. Pahwa, and S. Starrett, "Effective wind farm sizing method for weak power systems using critical modes of voltage instability," IEEE Trans. Power Syst., vol.27, no.3, pp. 1610-1617, 2012.

[3] E. Saiz-Marin, E. Lobato and I. Egido, "Local Hosting Capacity Increase by Means of Wind Farm Voltage Control Provision," IEEE Trans. Power Syst., vol. 29, pp. 1731-1738, 2014-01-01 2014

[4] I. Erlich, C. Feltes, and F. Shewarega, "Enhanced voltage drop control by VSC-HVDC systems for improving wind farm fault ride-through capability," IEEE Trans. Power Del., vol. 29, no. 1, pp. 378-385, 2014.
[5] V. S. S. Kumar, K. K. Reddy, and D. Thukaram, "Coordination of reactive power in grid-connected wind farms for voltage stability enhancement," IEEE Trans. Power Syst., vol. 29, no. 5, pp. 2381-2390, Sep. 2014.

[6] T. Ding, Q. Guo, H. Sun, et al. "A quadratic robust optimization model for automatic voltage control on wind farm side," in Proc. IEEE Power Energy Soc. Gen. Meeting (PES), Vancouver, BC, Canada, 2013, pp. 1-5.

[7] X. Zhu, Y. Wang and C. Fu," Strategy of reactive power and voltage control in large wind farms integrated region," Power System Technology, 2010 International Conference on, Hangzhou, 2010, pp. 1-5.

[8] Q. Wei, R.G. Harley, G.K. Venayagamoorthy, "Coordinated reactive power control of a large wind farm and a STATCOM using heuristic dynamic programming," IEEE Trans. Energy Conv., vol. 24, no. 2, pp. 493-503, June 2009.

[9] G. Tapia, A. Tapia, and J. X. Ostolaza, "Proportional-integral regulator-based approach to wind farm reactive power management for secondary voltage control," IEEE Trans. Power Syst., vol.22, no.2, pp. 488-498, June 2007.

[10] B. Rabelo, W. Hofmann, J. L. Silva, et al. "Reactive power control in doubly-fed induction generators for wind turbines," in Proc. IEEE Power Electronics Specialists Conf. PESC,Island of Rhodes, Greece, Jun. 15-19, 2008, pp. 106-112.

[11] P. Mitra, L. Zhang, and L. Harnefors, "Offshore wind integration to a weak grid by VSC-HVDC links using power-synchronization control: A case study," IEEE Trans. Power Del., vol. 29, no. 1, pp. 453-461, 2014.

[12] E. Vittal, M. O'Malley and A. Keane, "A steady-state voltage stability analysis of power systems with high penetrations of wind," IEEE Trans. Power Syst., vol. 25, pp. 433-442, 2010-01-01 2010.

[13] E. Vittal, A. Keane and M. O'Malley, "Varying penetration ratios of wind turbine technology for voltage and frequency stability," in Proc. IEEE PES General Meeting, Pittsburgh, USA, 2008.

[14] T. L. Ha, S. Santoso and Q. N. Thang, "Augmenting Wind Power Penetration and Grid Voltage Stability Limits Using ESS: Application Design, Sizing, and a Case Study," IEEE Trans. Power Syst., vol. 27, pp. 161-171, 2012.

[15] J. D. McCalley, S. Wang, Q. L. Zhao, and et al, "Security boundary visualization for systems operation," IEEE Trans. Power Syst., vol. 12, no.2, pp. 940-947, May 1997.

[16] J. F. Su, Y. X. Yu, H. J. Jia, and et al, "Visualization of voltage stability region of bulk power system," International Conference on Power System Technology, vol.3, pp. 1665-1668, Dec. 2002, China.

[17] W. Wei, P. Zhang, L. Min, and et al, "Voltage stability margin computation and visualization for Tristate South Colorado area using EPRI power system voltage stability region (PSVSR) program" Asia-Pacific Power and Energy Engineering Conference, pp. 1-6, Mar., 2009, China.

[18] Q. Guo, H. Sun, Y. Liu, et al. "Distributed Automatic Voltage Control framework for large-scale wind integration in China" in Power and Energy Society General Meeting, 2012 IEEE, San Diego, CA, 2012, pp. 1-5.

[19] Q. Guo, H. Sun, B. Wang, et al. "Hierarchical automatic voltage control for integration of large-scale wind power: design and implementation", Electric Power Systems Research, no. 120, pp. 234-241, 2015.

[20] T. Ding, Q. Guo, R. Bo, et al. "A static voltage security region for centralized wind power integration-Part I: Concept and method," Energies, vol. 7, no. 1, pp. 420-443, 2014.

[21] T. Ding, Q. Guo, R. Bo, et al. "A static voltage security region for centralized wind power integration-Part II: Applications," Energies, vol. 7, no. 1, pp. 444-461, 2014.

[22] T. Ding, R. Bo, H. Sun, et al. "A Robust Two-Level Coordinated Static Voltage Security Region for Centrally Integrated Wind Farms," IEEE Trans. Smart Grid, vol. pp, 2015, pp. 1-1.

[23] T. Niu, H. Liu, Q. Guo, et al, "Wind farm side optimal power flow based on DistFlow and SOCP: Model and case study," in Power and Energy Engineering Conference, 2014 IEEE PES Asia-Pacific, Kowloon, Hong Kong, 2014, pp. 1-5.

[24] Baran M E, Wu F. "Network reconfiguration in distribution systems for loss reduction and load balancing" IEEE Trans. Power Del., vol. 4, no. 2, pp. 1401-1407, 1989.

[25] Salih S N, Chen P. "On coordinated control of oltc and reactive power compensation for voltage regulation in distribution systems with wind power". IEEE Trans. Power Syst., pp.1-10, 2015 
[26] Daratha N, Das B, Sharma J. "Coordination between OLTC and SVC for voltage regulation in unbalanced distribution system distributed generation”, IEEE Trans. Power Del., vol. 29, no. 1, pp. 289-299, 2014.

[27] Viawan F A, Karlsson D. "Voltage and reactive power control in systems with synchronous machine-based distributed generation". IEEE Trans. on Power Del. vol. 23, no. 2, pp. 1079-1087, 2008.

[28] Ye R, Suganthan P N, Srikanth N. "A comparative study of empirical mode decomposition-based short-term wind speed forecasting methods". IEEE Trans. Sustainable Energy, vol. 6, no. 1, pp. 236-244, 2015.

[29] Singh, Rohan, Sahay, Kishan Bhushan, Srivastava, Shubhankar Aseet. "Short-term wind speed forecasting of Oak Park Weather Station by using different ANN algorithms" Smart Grid Technologies - Asia (ISGT ASIA), 2015 IEEE Innovative, 2015, pp. 1-6.

[30] Neumann, T.; Wijnhoven, T.; Deconinck, G.; Erlich, I "Enhanced Dynamic Voltage Control of Type 4 Wind Turbines During Unbalanced Grid Faults" IEEE Trans. Energy Conversion. vol. 30, no. 4, pp. 1650 1659,2015

[31] De Rijcke S, Ergun H, Van Hertem D, et al. "Grid Impact of Voltage Control and Reactive Power Support by Wind Turbines Equipped With Direct-Drive Synchronous Machines". IEEE Trans. Sustainable Energy, vol. 3, no. 4, pp. 890-898, 2012

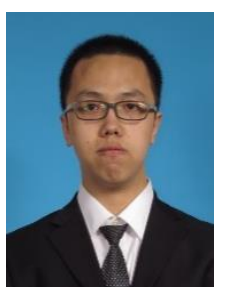

Tao Niu received his Bachelor degree from the Department of Electrical Engineering, Tsinghua University, Beijing, China, in 2014. He is pursuing the Ph.D. degree at Dept. of Electrical Engineering at Tsinghua University. His research interests include voltage security region, automatic reactive power voltage control and renewable generation integration.

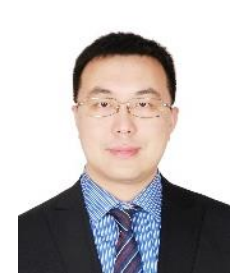

Qinglai Guo (SM'2014) was born in Jilin City, Jilin Province in China on Mar. 6, 1979. He graduated from the Department of Electrical Engineering, Tsinghua University, Beijing, China, in 2000 with B.S degree. He received his $\mathrm{PhD}$ degree from Tsinghua University in 2005 where he is now an associate professor. He is a member of CIGRE C2.13 Task Force on Voltage/Var support in System Operations. His special fields of interest include smart grids, cyber-physical systems and electrical power control center applications.

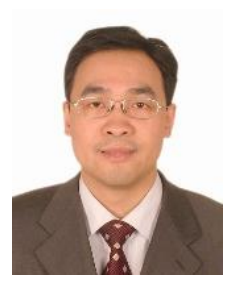

Hongbin Sun (SM' 2012) received his double B.S. degrees from Tsinghua University in 1992, the Ph.D from Dept. of E.E., Tsinghua University in 1997. He is now Changjiang Scholar of Education Ministry of China, full professor of electrical engineering in Tsinghua Univ. and assistant director of State Key Laboratory of Power Systems in China. From 2007.9 to 2008.9, he was a visiting professor with School of EECS at the Washington State University in Pullman. He is a Fellow of IET. He is a member of IEEE PES CAMS Cascading Failure Task Force and CIGRE C2.13 Task Force on Voltage/Var support in System Operations. In recent 15 years, he led a research group in Tsinghua University to develop a commercial system-wide automatic voltage control systems, which has been applied to PJM interconnection, the largest regional power grid in USA, and to more than 60 large-scale power grids in China. He published more than 300 academic papers. He won the China National Technology Innovation Award in 2008, the National Distinguished Teacher Award in China in 2009, and the National Science Fund for Distinguished Young Scholars of China in 2010. His research interests include smart grids, renewable generation integration, and electrical power control center applications.

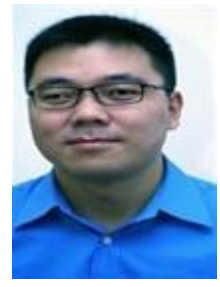

Qiuwei Wu (S'04-M'08-SM'15) received the B.Eng. and M.Eng. degrees in power system and automation from Nanjing University of Science and Technology, Nanjing, China, in 2000 and 2003, respectively, and the $\mathrm{Ph} . \mathrm{D}$. degree in power system engineering from Nanyang Technological University, Singapore, in 2009. He was a Senior R\&D Engineer with VESTAS Technology R\&D Singapore Pte Ltd., Singapore, from March 2008 to October 2009.

He was a Postdoc with the Centre for Electric Technology
(CET), Department of Electrical Engineering, Technical University of Denmark (DTU), Kgs. Lyngby, Denmark, from November 2009 to October 2010, an Assistant Professor from November 2010 to August 2013, and has been an Associate Professor since September 2013 with the same centre.

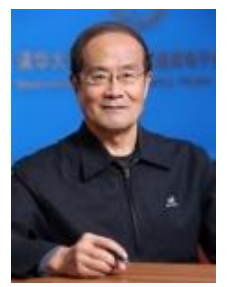

Boming Zhang (SM'95-F'10) received the Ph.D. degree in electrical engineering from Tsinghua University, Beijing, China, in 1985. Since 1985, he has been with the Electrical Engineering Department, Tsinghua University, promoted to a Professor in 1993. His interest is in power system analysis and control, especially in the EMS advanced applications in the Electric Power Control Center (EPCC).

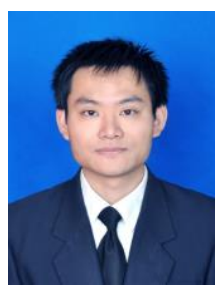

Tao Ding (S'13-M'15) received the degree in mathematics from Southeast University, Nanjing, China, the B.S.E.E. and M.S.E.E. degrees from Southeast University, Nanjing, China, and the Ph.D. degree from Tsinghua University, Beijing, China, in 2007, 2009, 2012 and 2015, respectively. From 2013 to 2014, he was a Visiting Scholar at the Department of Electrical Engineering and Computer Science, University of Tennessee, Knoxville, TN, USA. He is currently an Associate Professor with the State Key Laboratory of Electrical Insulation and Power Equipment and the School of Electrical Engineering, Xi' an Jiaotong University, Xi'an, China. His research interests include electricity markets, power system economics and optimization methods, and power system planning and reliability evaluation. He was the recipient of the Outstanding Graduate Award of Beijing City. 